\title{
Subsurface structure and magnetic parameters of Fe-Mo-Cu-B metallic glass
}

\author{
Marcel Miglierini, \\ Mariusz Hasiak, \\ Marek Bujdoš
}

\begin{abstract}
Subsurface properties of ${ }^{57} \mathrm{Fe}_{81} \mathrm{Mo}_{9} \mathrm{Cu}_{1} \mathrm{~B}_{9}$ metallic glass were studied by conversion electron and conversion X-ray Mössbauer spectrometry. They were applied to both surfaces of the ribbons. Deviations in structural surface features are exhibited via different contents of crystalline phases, which were identified as bcc-Fe and magnetite. The presence of small ferromagnetic particles was also suggested from magnetic measurements. An influence of irradiation with $130-\mathrm{keV} \mathrm{N}^{+}$ions on surface properties of the as-quenched alloy is also discussed.
\end{abstract}

Key words: ion bombardment $\bullet$ metallic glasses $\bullet$ Mössbauer spectrometry $\bullet$ soft magnetic properties $\bullet$ surface structure

\section{Miglierini ${ }^{凶}$}

Institute of Nuclear and Physical Engineering,

Faculty of Electrical Engineering and Information

Technology,

Slovak University of Technology in Bratislava,

Ilkovičova 3, 81219 Bratislava, Slovakia

and Department of Nuclear Reactors,

Faculty of Nuclear Sciences and Physical Engineering,

Czech Technical University in Prague,

V Holešovičkách 2, 18000 Prague 8,

Czech Republic

E-mail: marcel.miglerini@stuba.sk

\section{Hasiak}

Department of Mechanics and Materials Science,

Wrocław University of Technology,

25 Smoluchowskiego Str., 50-370 Wrocław, Poland

\section{Bujdoš}

Institute of Laboratory Research on Geomaterials,

Faculty of Natural Sciences,

Comenius University,

Mlynská dolina, 84215 Bratislava, Slovakia

Received: 18 June 2014

Accepted: 12 October 2014

\section{Introduction}

Recently, Fe-based metallic glasses (MGs) have been suggested as candidates for magnetic cores of accelerator radio-frequency (RF) cavities to improve their performance [1]. For this particular application, they are exposed to ion irradiation caused by beam losses. During the machine operation, all construction materials are exposed to radiation caused by lost beam particles. The lost primary beam particles interact with the material of the beam-pipe walls and produce secondary particles, such as light fragments and neutrons. At high beam intensities, even low-level beam losses may cause high radiation load.

Originally, it was expected that MGs should resist the radiation damage due to their homogeneous disordered structure [2]. They were considered as appropriate materials in radiation-exposed environments. However, results obtained during the last two decades pointed out that irradiation can induce changes of properties in MGs.

Pronounced changes in magnetic hyperfine interactions were revealed by irradiation of a $\mathrm{Fe}_{90} \mathrm{Zr}_{10}$ amorphous alloy with $209 \mathrm{MeV}{ }^{84} \mathrm{Kr}$ ions with the fluence of $3 \times 10^{14}$ ions $/ \mathrm{cm}^{2}$ [3]. The modification in the magnetic anisotropy introduced by swift heavy ion irradiation has been investigated in ferromagnetic MGs $\mathrm{Fe}_{78} \mathrm{~B}_{13} \mathrm{Si}_{9}$ and $\mathrm{Fe}_{40} \mathrm{Ni}_{38} \mathrm{Mo}_{4} \mathrm{~B}_{18}$ irradiated 
with $100 \mathrm{MeV}{ }^{127} \mathrm{I}$ ions at different fluences from $5 \times 10^{12}$ to $7.5 \times 10^{13}$ ions $/ \mathrm{cm}^{2}$ [4]. The magnetic anisotropy has been studied by measuring the relative line intensity ratio of Mössbauer sextets.

Irradiation with $120-\mathrm{MeV} \mathrm{Ag}$ and $350-\mathrm{MeV} \mathrm{Au}$ ions of $\mathrm{Fe}_{0.85} \mathrm{~N}_{0.15}$ and $\mathrm{Fe}_{73.9} \mathrm{Cu}_{0.9} \mathrm{Nb}_{3.1} \mathrm{Si}_{13.2} \mathrm{~B}_{8.9}$ amorphous films resulted in gradual removal of anisotropy and a decrease in coercivity, which was attributed to relaxation of internal stresses [5]. This demonstrates that swift heavy ions can be used for controlled modification of magnetic properties of thin films. At the fluence of $5 \times 10^{13} \mathrm{ions} / \mathrm{cm}^{2}$, the in-plane anisotropy completely disappeared.

In general, it can be concluded that ion irradiation of MGs may influence their thermal stability, crystallization processes, magnetic anisotropy, shapes of hysteresis loops, Curie temperatures, surface crystallization, and others.

A majority of investigation deals with bulk properties of MGs. However, due to apparent differences of the surfaces, which stem from the production process via rapid quenching on a rotating wheel, differences both in structure and hyperfine magnetic parameters are expected [6]. One of the main goals of this study is closer inspection of surfaces of NANOPERM-type MG. Surface regions can be effectively studied to the depth of about $200 \mathrm{~nm}$ and slightly less than $10 \mu \mathrm{m}$ by conversion electron Mössbauer spectrometry (CEMS) and conversion X-ray Mössbauer spectrometry (CXMS), respectively. At the same time, we are interested in the impact of microstructure on magnetic properties of the investigated alloys.

\section{Experimental details}

Ribbons of ${ }^{57} \mathrm{Fe}_{81} \mathrm{Mo}_{9} \mathrm{Cu}_{1} \mathrm{~B}_{9}$ MG were prepared by the method of rapid quenching. The casting was done in the air, that is, no protective atmosphere was used. The width and the thickness of the ribbons were about $1 \mathrm{~mm}$ and $20 \mu \mathrm{m}$, respectively. Their chemical composition was checked by optical emission spectrometry with inductively coupled plasma (Mo and B) and flame atomic absorption spectrometry $(\mathrm{Fe}$ and $\mathrm{Cu})$. As-quenched and partially crystallized samples were studied by CEMS and CXMS. For production of our sample, we have used iron enriched to about $50 \%$ in the ${ }^{57} \mathrm{Fe}$ isotope that facilitates the use of the CEMS and CXMS techniques.

Mössbauer effect experiments were performed with a constant acceleration spectrometer using a ${ }^{57} \mathrm{Co} / \mathrm{Rh}$ source. Calibration was done using a thin $(12.5 \mu \mathrm{m}) \alpha$-Fe foil at room temperature. Evaluation of the spectra was accomplished by the Confit software [7].

Measurements of soft magnetic properties comprising hysteresis loops and temperature dependence of magnetization were carried out by a VersaLab (Quantum Design) system in a wide temperature range using different external magnetic fields.

As-quenched alloys were irradiated by $130-\mathrm{keV}$ nitrogen ions to the air side of the ribbons, that is, the ones that were in contact with the surrounding atmosphere during the rapid quenching process of their preparation. The irradiation was performed using a cascade accelerator at the Slovak University of Technology [8]. The parameters of the particle beams and their ranges were calculated [9] using the SRIM2008 and $\mathrm{S}^{3} \mathrm{M}$ codes.

\section{Results and discussion}

The inspection of the as-quenched ribbons of the ${ }^{57} \mathrm{Fe}_{81} \mathrm{Mo}_{9} \mathrm{Cu}_{1} \mathrm{~B}_{9}$ MG revealed traces of crystallization at their both sides. The wheel side of the ribbon refers to the side that was in direct contact with the quenching wheel during the production, whereas the air side was exposed to the surrounding atmosphere. As demonstrated by Fig. 1, quite a remarkable amount of bcc-Fe and magnetite crystalline phases is found, namely, at the wheel side of the ribbons in near-surface regions (down to the depth of about $200 \mathrm{~nm}$ ) that were scanned by conversion electrons (CEMS). The corresponding Mössbauer subspectra of magnetite are green (A-sites) and dark green (B-sites), whereas those of bcc-Fe nanocrystals are blue. Broad cyan components represent $\mathrm{Fe}$ atoms located at the surfaces of the nanograins, the so-called interface regions [10]. The relative contents of bcc-Fe (including the interface regions) and magnetite are of $12 \%$ and $16 \%$, respectively. The deeper subsurface regions that were examined by conversion X-rays (CXMS) show only traces of bcc-Fe. This indicates that magnetite resides mostly inside the surface imperfections (cavities) that are observed at this side of the ribbons and which are caused by small gas bubbles trapped between the wheel and the quenching melt during the production. In addition, surface roughness of the wheel can also be stamped into the ribbon's exterior.

On the other hand, the air side, which is visually shiny, exhibits a smooth surface without any obvious imperfections. Consequently, only very tiny amounts of bcc-Fe crystallites are found in the shallow subsurface layers as seen from the respective CEMS spectrum in Fig. 1. It is noteworthy that no traces of crystallization were identified in deeper subsurface regions by CXMS.

Apart from the crystalline subspectra, all Mössbauer spectra in Fig. 1 show a rather broad central doublet. The latter can be ascribed to the amorphous matrix of the investigated alloy that is paramagnetic at room temperature. This behavior is confirmed by measurements of magnetic properties. For example, the normalized magnetization plotted against temperature in Fig. 2 for the as-quenched sample falls to low values at room temperature $(300 \mathrm{~K})$ but not to zero. The magnetization data were acquired in the field of $10 \mathrm{mT}$. This means that the alloy should contain some amount of ferro/ferrimagnetic phases with high Curie points. Using the results of Mössbauer spectrometry, these magnetically active phases were shown to be bcc-Fe and magnetite that are, moreover, located only at the surface regions.

After annealing at $370^{\circ} \mathrm{C}$ and $450^{\circ} \mathrm{C}$, an increased contribution of ferromagnetic nanocrystalline phases is expected. This is demonstrated by still higher 

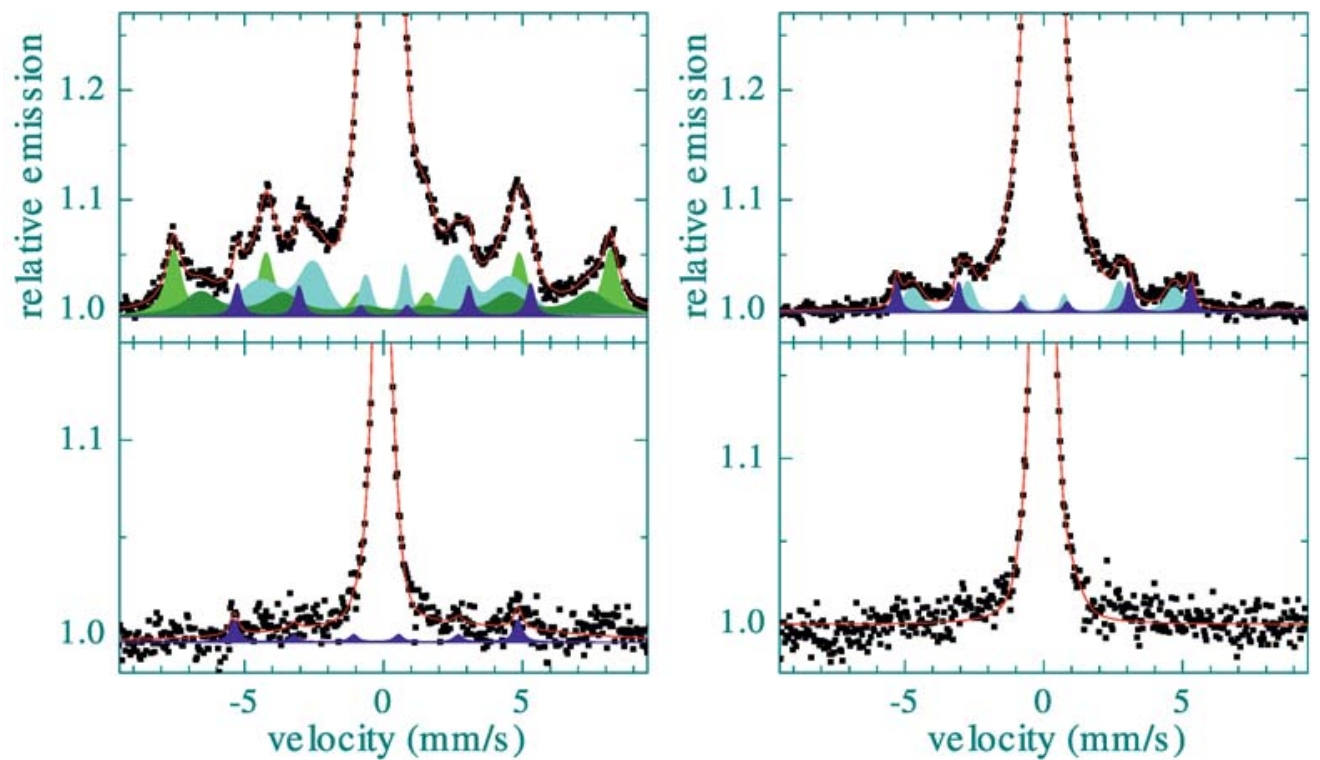

Fig. 1. Mössbauer spectra and their crystalline components (see text for details) recorded by CEMS (top) and CXMS (bottom) techniques from the wheel (left) and air (right) sides of the ${ }^{57} \mathrm{Fe}_{{ }_{81}} \mathrm{Mo}_{9} \mathrm{Cu}_{1} \mathrm{~B}_{9}$ metallic glass ribbons in the as-quenched states.

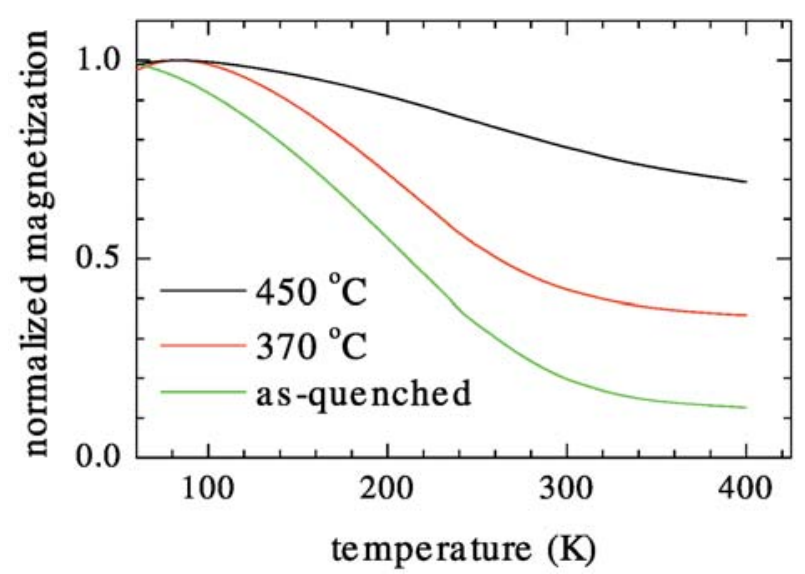

Fig. 2. Normalized magnetization plotted vs. temperature for a ${ }^{57} \mathrm{Fe}_{81} \mathrm{Mo}_{9} \mathrm{Cu}_{1} \mathrm{~B}_{9}$ alloy in an as-quenched state and after annealing at $370^{\circ} \mathrm{C}$ and $450^{\circ} \mathrm{C}$.

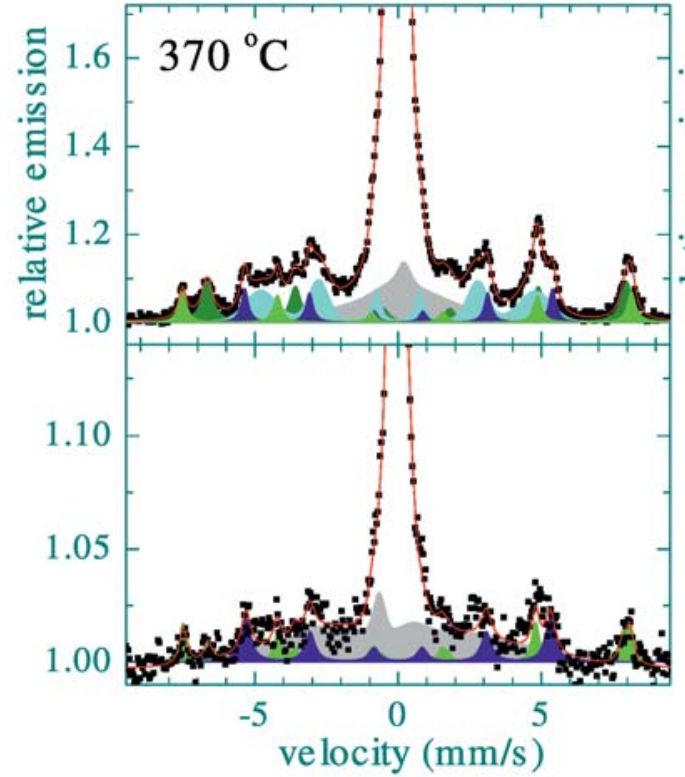

values of normalized magnetization toward higher measuring temperatures in Fig. 2.

Mössbauer spectrometry experiments confirm this assumption. In Fig. 3, we present the CEMS and CXMS spectra taken from the wheel sides of the annealed ribbons. In contrast to the as-quenched alloy, the presence of magnetite is observed also in deeper regions of the ribbons as seen from the corresponding CXMS spectra. Their content increases with temperature of annealing. More importantly, higher contents of ferromagnetic crystallites (both bcc-Fe and magnetite) polarized the originally paramagnetic amorphous matrix. As a consequence, some of the amorphous regions exhibit weak magnetic interactions that were refined during the fitting procedure by distributed magnetic sextets. They are shown in Fig. 3 by light grey broadened spectral components. Possible superparamagnetic

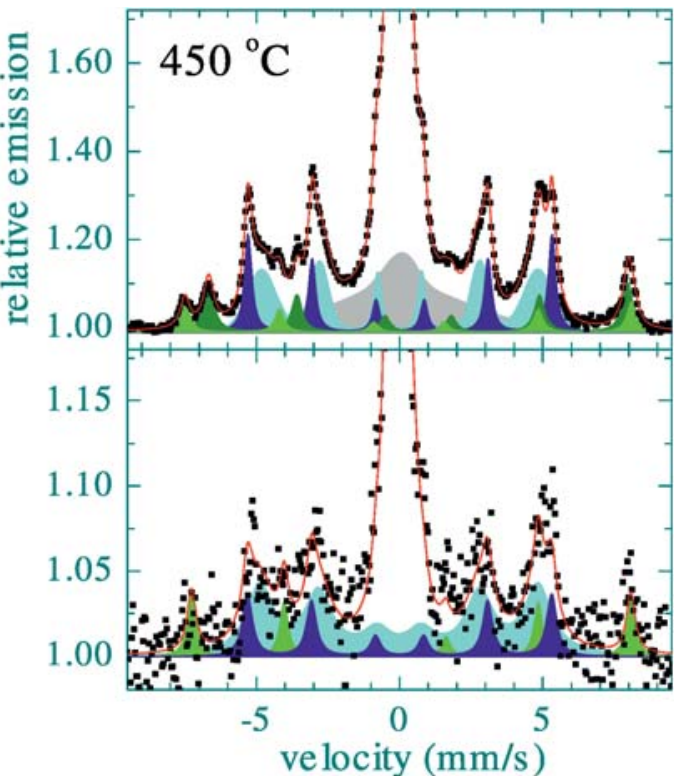

Fig. 3. Mössbauer spectra and their crystalline components recorded by CEMS (top) and CXMS (bottom) techniques from the wheel sides of the ${ }^{57} \mathrm{Fe}_{81} \mathrm{Mo}_{9} \mathrm{Cu}_{1} \mathrm{~B}_{9}$ metallic glass ribbons annealed at $370^{\circ} \mathrm{C}$ (left) and $450^{\circ} \mathrm{C}$ (right). 


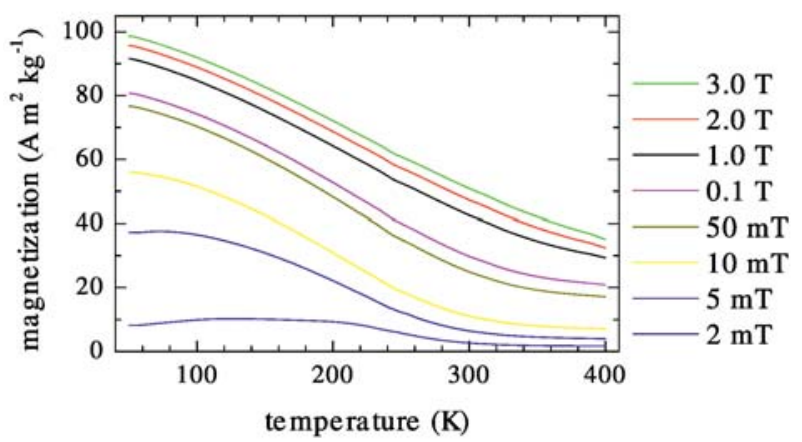

Fig. 4. Hysteresis loops measured at temperatures from $50 \mathrm{~K}$ to $400 \mathrm{~K}(\Delta T=50 \mathrm{~K})$ on an as-quenched ${ }^{57} \mathrm{Fe}_{81} \mathrm{Mo}_{9} \mathrm{Cu}_{1} \mathrm{~B}_{9}$ alloy.

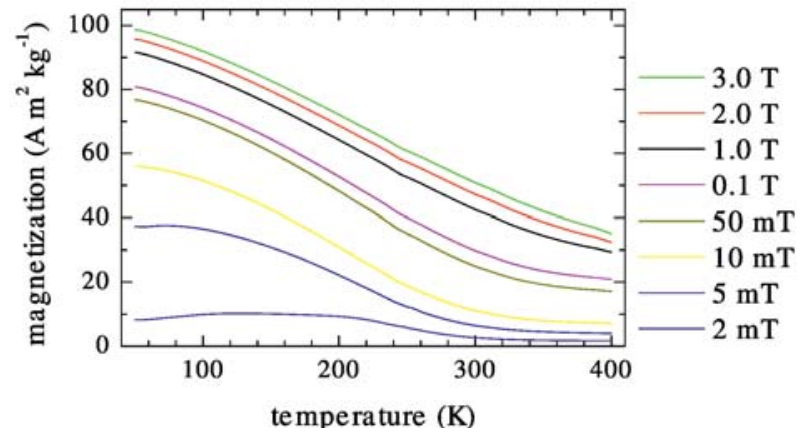

Fig. 5. Magnetization plotted versus temperature for a ${ }^{57} \mathrm{Fe}_{81} \mathrm{Mo}_{9} \mathrm{Cu}_{1} \mathrm{~B}_{9}$ alloy in an as-quenched state, measured at specified external magnetic fields.

relaxation of the fine ferromagnetic particles would be demonstrated by their rather broad spectral lines [10], which is, however, not the present case.

Examples of isothermal hysteresis loop measurements performed in a temperature range from $50 \mathrm{~K}$ to $400 \mathrm{~K}(\Delta T=50 \mathrm{~K})$ are shown in Fig. 4 for an as-quenched alloy. The same soft magnetic behavior was also observed in the annealed samples. Moreover, the decrease of maximum magnetization with temperature observed from the hysteresis loops was also demonstrated in Fig. 2 with the help of

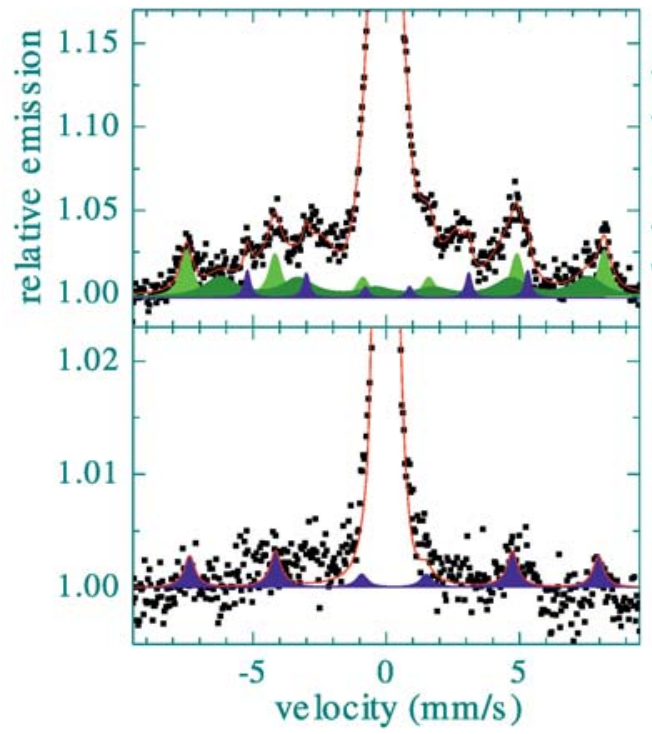

normalized magnetization. The latter shows only the tendency of the temperature behavior of the magnetization, whereas from Fig. 4, the absolute values of maximum magnetization can be derived.

The effect of external magnetic field on the temperature dependence of magnetization for the as-quenched alloy is shown in Fig. 5. An increase of magnetization with the applied magnetic field is observed that is a typical behavior of ferromagnetic materials.

Mössbauer spectra of the as-quenched alloys that were exposed to irradiation with $130-\mathrm{keV}$ nitrogen ions to the total dose of $2.5 \times 10^{17} \mathrm{~N}^{+} / \mathrm{cm}^{2}$ are presented in Fig. 6 . The maximum of the penetration profile was calculated using the SRIM code to be at the depth of $147 \mathrm{~nm}$. Because the irradiation was accomplished on the air side of the ribbons, we do not expect any changes at the wheel side of a 20- $\mu$ m-thick ribbon. Indeed, as seen in Fig. 6 left, both CEMS and CXMS spectra are similar to those of the non-irradiated as-quenched alloy from Fig. 1.

A drastic effect of ion irradiation was found at the air side. A remarkable increase in the bcc-Fe content is identified, which can be a consequence of a local heating during the irradiation even though the whole material was cooled by vapors of liquid nitrogen. Nevertheless, possible formation of nitrides, which is indicated by additional spectral lines, is clearly visible.

\section{Conclusions}

Surface properties of a NANOPERM-type ${ }^{57} \mathrm{Fe}_{81} \mathrm{Mo}_{9} \mathrm{Cu}_{1} \mathrm{~B}_{9}$ alloy were investigated with the help of CEMS and CXMS techniques that provide information from the depths of about $200 \mathrm{~nm}$ and less than $10 \mu \mathrm{m}$, respectively. Formation of bcc-Fe and magnetite crystalline phases was revealed in the as-quenched alloy, especially on the wheel side and close to the surface by CEMS. In deeper layers, CXMS unveiled only a small amount of crystalline

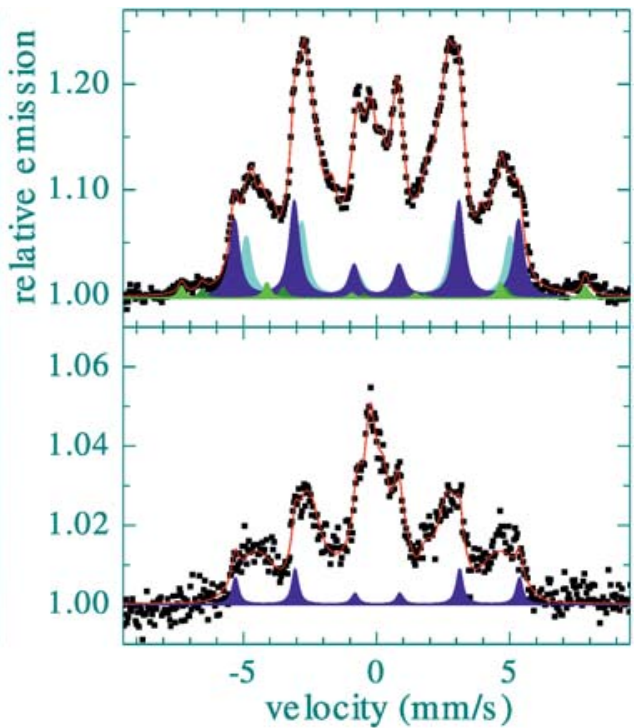

Fig. 6. Mössbauer spectra and their crystalline components recorded by CEMS (top) and CXMS (bottom) techniques from the wheel (left) and air (right) sides of the ${ }^{57} \mathrm{Fe}_{81} \mathrm{Mo}_{9} \mathrm{Cu}_{1} \mathrm{~B}_{9}$ metallic glass ribbons in the as-quenched states after ion irradiation with the dose of $2.5 \times 10^{17} \mathrm{~N}^{+} / \mathrm{cm}^{2}$. 
bcc-Fe phase. The same small contribution of crystalline bcc-Fe is seen at the air side by CEMS while no traces of crystalline phases are found by CXMS.

Magnetic measurements indicated the presence of some contents of ferromagnetic phases. These were actually confirmed by the aforementioned results of Mössbauer spectrometry.

Irradiation with $130-\mathrm{keV} \mathrm{N}^{+}$ions affected mostly the side of the ribbon through which they penetrated into the material (i.e., the air side). The opposite (wheel) side was practically unchanged.

Acknowledgment. This work was supported by the research grants GAČR 14-12449S, SK-PL-0032-12, and VEGA 1/0286/12.

\section{References}

1. Spiller, P., \& Franchetti, G. (2006). The FAIR accelerator project at GSI. Nucl. Instrum. Methods A, 561, 305-309. DOI: 10.1016/j.nima.2006.01.043.

2. Spiller, P., Blasche, K., Franczak, B., Kirk, M., Huelsmann, P., Omet, C., Ratschow, S., \& Stadlmann, J. (2005). Accelerator plans at GSI for plasma physics applications. Nucl. Instrum. Methods A, 544, 117-124. DOI: 10.1016/j.nima.2005.01.289.

3. Kuzmann, E., Lakatos-Varsanyi, M., Nomura, K., Ujihira, Y., Masumoto, T., Principi, G., Tosello, C., Havancsak, K., \& Vertes, A. (2000). Combination of electrochemical hydrogenation and Mössbauer spectroscopy as a tool to show the radiation effect of energetic heavy ions in $\mathrm{Fe}-\mathrm{Zr}$ amorphous alloys. Electrochem. Commun., 2, 130-134. DOI: 10.1016/ S1388-2481(99)00164-2.

4. Amrute, K. V., Nagare, B. J., Fernandes, R. P., Sivakumar, V. V., Gupta, A., Kanjilal, D., \& Kothari, D. C. (2005). Modification of magnetic anisotropy in ferromagnetic metallic glasses using high energy ion beam irradiation. Surf. Coat. Technol., 196, 135-138. DOI: 10.1016/j.surfcoat.2004.08.089.

5. Dubey, R., Gupta, A., Sharma, P., Darowski, N., \& Schumacher, G. (2007). Tailoring of magnetic anisotropy in amorphous and nanocrystalline soft magnetic alloys using swift heavy ions. J. Magn. Magn. Mater., 310, 2491-2493. DOI: 10.1016/j.jmmm.2006.11.091.

6. Miglierini, M., Hatala, T., Frydrych, J., \& Šafářová, K. (2012). Surface crystallization of Co-containing NANOPERM-type alloys. Hyperfine Interact., 205, 125-128. DOI: 10.1007/s10751-011-0495-1.

7. Žák, T., \& Jirásková, Y. (2006). CONFIT: Mössbauer spectra fitting program. Surf. Interface Anal., 38, 710-714. DOI: 10.1002/sia.2285.

8. Kovác, P., Pavlovic, M., \& Dobrovodsky, J. (1994). A $0.9 \mathrm{MV}$ accelerator for materials research at the STU Bratislava. Nucl. Instrum. Methods Phys. Res. Sect. B-Beam Interact. Mater. Atoms, 85, 749-751. DOI: 10.1016/0168-583X(94)95916-1.

9. Pavlovic, M., \& Strašík, I. (2007). Supporting routines for the SRIM code. Nucl. Instrum. Methods Phys. Res. Sect. B-Beam Interact. Mater. Atoms, 257, 601-604. DOI: 10.1016/j.nimb.2007.01.047.

10. Miglierini, M., \& Grenèche, J. -M. (2003). Temperature behaviour of iron nanograins in NANOPERM-type alloys. J. Phys.-Condens. Matter, 15, 5637-5648. DOI: 10.1088/0953-8984/15/32/323. 